\title{
Spectral libraries and their uncertainties
}

\author{
P. Coelho \\ Institut d'Astrophysique, 98 bis Bd Arago, 75014 Paris, France
}

\begin{abstract}
Libraries of stellar spectra are fundamental tools in the study of stellar populations and in automatic determination of atmospheric parameters for large samples of observed stars. In the context of the present volume, here I give an overview of the current status of stellar spectral libraries from the perspective of stellar population modeling: what we have currently available, how good they are, and where we need further improvement.
\end{abstract}

Keywords: stellar spectral libraries, stellar atmospheres, atmospheric parameters

PACS: 97.10.Ex

\section{INTRODUCTION}

An evolutionary stellar population model [e.g. 1] has two main ingredients: a set of stellar evolutionary models (tracks or isochrones) that predicts how the stars are distributed in the HR diagram, and a library of stellar observables (e.g. colours, spectra, spectral indices) that is used to predict the colours or spectra of a stellar population, given the evolutionary predictions (see articles by A. Vazdekis and S. Cassissi in this volume).

This article focus on the library of stellar observables, more specifically, on libraries of stellar spectra. A good stellar library is at the very heart of accurate SP models, and should ideally provide complete coverage of the HR diagram, accurate atmospheric parameters (effective temperature $\mathrm{T}_{\text {eff }}$, surface gravities $\log g$, metallicities $\mathrm{Z}$ and abundances $[\mathrm{Fe} / \mathrm{H}],[\mathrm{Mg} / \mathrm{Fe}]$, etc.), and good wavelength coverage and/or good spectral resolution, depending on the aimed application. Either empirical or theoretical libraries can be used in stellar population (SP) modeling and both types have improved dramatically in recent years, allowing the construction of more accurate SP models. There are plenty of models in literature using both empirical (e.g. [2, 3], the visible range of [4]) and theoretical libraries (e.g. [5,6]). Observations are also becoming increasingly better in terms of spectral resolution and coverage and demanding more from the modelling point of view [e.g. 7].

A good starting point to inspect the libraries available in literature is the comprehensive list maintained by D. Montes 1 , with more than 80 libraries currently listed. The libraries cover a virtually complete wavelength range with theoretical libraries, and from near UV to K-band with empirical ones, at spectral resolutions $R=\Delta \lambda / \lambda$ from 200 to 80000 (even higher for a few selected stars), and different coverages of atmospheric parameters and abundances. In the following sections I highlight the strengths and caveats of empirical and theoretical libraries currently available.

\footnotetext{
1 http://www.ucm.es/info/Astrof/invest/actividad/spectra.html
} 


\section{EMPIRICAL LIBRARIES}

An empirical library is an homogeneous compilation of observed stellar spectra. It is not a simple task to assemble a library that simultaneously features high S/N, good flux calibration, large wavelength coverage, high spectral resolution and accurately derived stellar parameters. Major improvements have been made in the last years, with the publications of empirical libraries with improved spectral resolution and parameter coverage: e.g. STELIB [8], UVES POP [9], Indo-US [10], ELODIE [11, 12], MILES $[13,14]$. Being based on real stars, the major advantage of an empirical library is that the spectral properties are highly reliable, limited only by the quality of the observations. High quality observations are limited to the closest stars, and thus the coverage of the HR diagram and abundances are biased towards the typical stellar population targeted by the observations.

The coverage of two of the most complete libraries available nowadays, ELODIE and MILES, are shown in Fig. 1 in $\mathrm{T}_{\text {eff }}$ vs. $\log g$ space. ELODIE 2 in its current version (3.1) contains 1388 starts, covers the wavelength range from 4000 to $6800 \AA$, and has a typical S/N of 150 per pixel at $5550 \AA$. Although it has a somewhat limited wavelength coverage, it has very high spectral resolution $(\mathrm{R}=10000$ for flux calibrated spectra and $\mathrm{R}=42000$ for flux normalised to the pseudo-continuum). MILES 3 was assembled trying to fill major gaps that existed in previous empirical libraries in terms of HR coverage. It has 985 stars with spectra ranging from 3525 to $7500 \AA$ at a $2.3 \AA$ (FWHM) resolution. Stars from ELODIE and MILES are shown in the upper and lower panels in Fig. 1 respectively, for three bins of [Fe/H] as indicated. Isochrones from [15] for ages 30Myr, $100 \mathrm{Myr}$, 1Gyr and 10Gyr are overplotted. Clearly the bin with the iron abundance around the solar value is the most complete. A possible caveat in this regime is that the most luminous giants in the upper part of the red giant branch and asymptotic giant branch evolutionary phases are not yet being covered, but this should not have a large effect modeling the visible wavelength range. Empirical libraries that focus specifically on those stars [e.g. 16] may be used to cover these phases if needed. Outside the solar metallicity regime, the coverage is less complete: in the super-solar regime, populations older than $\sim 100$ Myr can still be modeled reliably, and in the case of metal poor populations $([\mathrm{Fe} / \mathrm{H}] \sim-1.8)$, only old populations $(\sim 10 \mathrm{Gyr})$ can be modeled.

The main caveat of empirical libraries is that SP models based solely on them are not able to reproduce consistently the medium to high resolution spectral features of systems which have undergone a star formation history different than the solar-neighborhood. The first compelling evidence of this limitation was presented by [17] (see also article by R. Peletier in this volume), who showed that SP models for Lick/IDS indices cannot reproduce the indices measured in elliptical galaxies, indicating that these systems are overabundant in $\alpha$-elements relative to the Sun. This happens because, by construction, the abundance pattern of models based on empirical libraries is dictated by that of the library stars, which mirrors the abundance pattern mainly of the solar neighborhood [e.g. 18]. Theoretical libraries must be used to overcome this limitation, either solely or

2 http://www.obs.u-bordeauxl.fr/m2a/soubiran/elodie_library.html

3 http://www.ucm.es/info/Astrof/miles/miles.html 

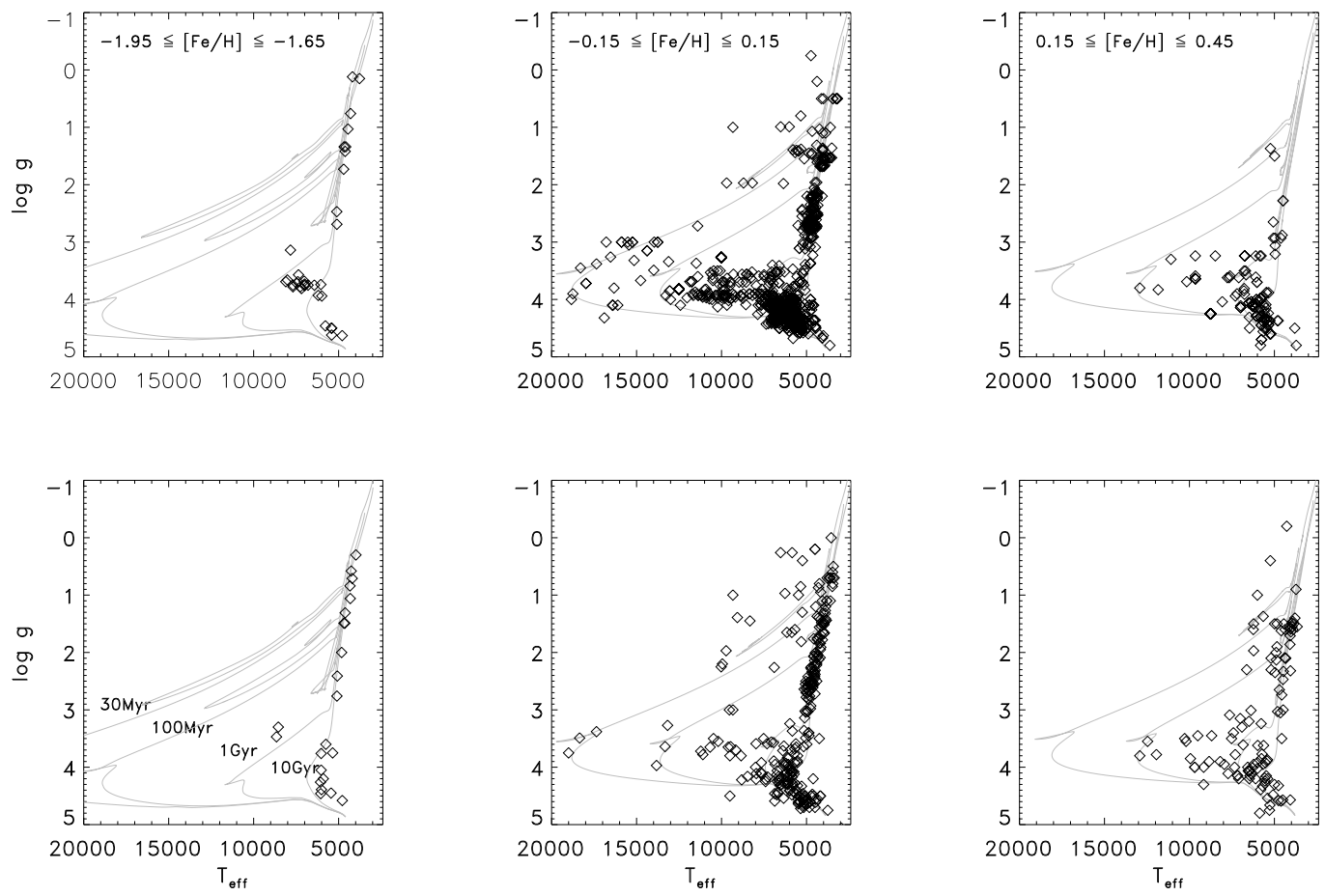

FIGURE 1. Coverage of ELODIE and MILES stellar libraries (top and bottom rows, respectively) in $\mathrm{T}_{\text {eff }}$ vs. $\log g$ space. Three bins of $[\mathrm{Fe} / \mathrm{H}]$ are shown, as indicated in the top figures. Isochrones from [15] are overplotted, for ages $30 \mathrm{Myr}, 100 \mathrm{Myr}, 1 \mathrm{Gyr}$ and $10 \mathrm{Gyr}$. The abundance $[\mathrm{Fe} / \mathrm{H}]$ of the isochrones are $-1.75,+0.06$ and $0.25 \mathrm{dex}$ for the left-hand, central and right-hand panels respectively.

in combination with empirical libraries [see e.g. 19].

\section{THEORETICAL LIBRARIES}

A theoretical (or synthetic) spectral library is based on model atmospheres predictions and atomic and molecular line lists. A model atmosphere is the run of temperature, pressure (gas, electron and radiation), convective velocity and flux, and more generally of all relevant quantities as a function of some depth variable (geometrical, optical depth at some special frequency, or column mass). The synthetic spectrum or flux distribution is the emergent flux computed based on a model atmosphere and atomic and molecular line opacity lists, and is required for comparison with observations. Theoretical libraries have the advantage of covering the parameter space in $\mathrm{T}_{\text {eff }}, \log g$, and abundances at will. Moreover, a synthetic star has very well defined atmospheric parameters, infinite $\mathrm{S} / \mathrm{N}$, and covers larger wavelength ranges at higher resolutions than observed spectra. To compute a large synthetic library can be demanding in terms of computational time, but it is usually feasible. The caveat of theoretical libraries is that, being based on our knowledge of the physics of stellar atmospheres and databases of atomic and molecular transitions, those libraries are limited by the approximations and (in)accuracies of their 
underlying models and input data.

The ability of theoretical libraries in predicting broad-band colors and medium to high resolution spectral features has been assessed by e.g. [20, 21, 22, 23]. Studying libraries with large coverages in stellar atmospheric parameters, [22] finds that in general current models are able to reproduce stellar colors with accuracy for a fair interval in effective temperatures and gravities, but there are still some problems with $U-B$ and $B-V$ colors, and very cool stars in general $\left(\mathrm{T}_{\mathrm{eff}}<4000 \mathrm{~K}\right)$. Studying models for red giants, [21] finds that theoretical $\mathrm{T}_{\text {eff }}$ vs. color relations for visible and near-infrared colors agree with observations within $100 \mathrm{~K}$ down to $\mathrm{T}_{\text {eff }} \sim 3400 \mathrm{~K}$, but none of the existing theoretical relations reproduce the data below $\sim 3800 \mathrm{~K}$ in $U-B$ and $B-V$.

Concerning medium and high resolution features, [22] analyzed the performance of recent high-resolution libraries by comparing their predictions of spectral indices (from 3500 to $8700 \mathrm{~A}$ ) to measurements from empirical libraries. Here also it is found that many indices are well reproduced for a fair range of temperatures, but that lists of atomic and molecular opacities still need improvement in the blue region of the spectrum and for the cool stars regime in general. This is illustrated in Fig. 2, where predictions of the theoretical library by Coelho et al. [24] are plotted against observed values from MILES library, for six spectral indices. In general the agreement is good, but deviations appear in the cool stars regime (as easily seen for the indices $\mathrm{H} \gamma, \mathrm{G} 4300$ and Ca4455). Studying the performance of theoretical spectra in reproducing high resolution observations, [23] compared model predictions to three outstanding reference templates, namely the Sun, Arcturus and Vega. These authors fitted the high-resolution $(\mathrm{R}=522000)$ spectrum of the Sun in the region 3500 to $7000 \AA$ and found that the observed solar flux is reproduced within $9 \%$ in relative flux uncertainty (rms). Downgrading the spectrum to $\mathrm{R}=10^{5}$ brings the rms down to 5\%. The agreement between model and observations is better for Vega (1\%), and worse for Arcturus (9\%) at the same $\mathrm{R}=10^{5}$ resolution. They further compared the theoretical predictions with a selected sample of observations from ELODIE at $\mathrm{R}=42000$, and found that there is a trend in temperature and gravity: hotter stars are better reproduced than cooler ones, and dwarfs are better reproduced than giants. No trend with metallicity was found.

The origin of the deficiencies in the theoretical predictions may be two-fold: the underlaying physics theory and/or the input physics data (atomic and molecular opacities) of the models. In terms of physics theory, most of the libraries computed to cover large parameter spaces are computed under 1D and LTE assumptions. For stars of spectral types from $A$ to $G$ these approximations are in general suitable, but for other spectral types it is known that effects of 3D hydrodynamics, N-LTE, winds and chromospheric contribution may be important. In the regime of cool stars effects such as convection, variability, mass loss and dust formation become increasingly important [e.g. 25, 26, 27]. State-of-the-art hydrodynamical models are an important recent advancement [e.g. 28, 29], but these models are considerably time-consuming and until now no extensive grids of 3D models exist. In the hot stars regime, models taking into account N-LTE effects are mature and grids of N-LTE line-blanketed model atmospheres and fluxes of Oand B-type stars are available in literature [e.g. 30, 31, 32]. The modeling of mass loss and winds (particularly important in the UV wavelength range) is less well established (C. Leitherer, priv. comm.), but progress is being made [e.g. 33, 34].

In terms of physics basic data, databases of atomic and molecular transitions provide 
fewer lines with highly accurate oscillator strengths and broadening parameters than we would like, besides being often incomplete [see e.g. 35]. Constant updates and improvements are necessary from laboratory data, critical compilations, empirical adjustments and improved quantum mechanics computations [e.g. 36, 37, 38]. Some libraries of theoretical spectra opt for including atomic and molecular line lists which are as complete as possible, thus providing a good treatment of line-blanketing (i.e. include the so-called 'predicted lines', see [39]). These libraries are good for spectrophotometric predictions and low-spectral resolution studies [e.g. 40, 41, 42, 43]. But most of the predicted lines does not have accurate wavelengths and oscillator strengths, and thus are not appropriate to reproduce high spectral resolution features. Therefore other theoretical libraries were computed with shorter, fine-tuned, empirically calibrated atomic and molecular line lists [e.g. 44, 45, 24]. These libraries are much more accurate in reproducing high-resolution features, but are not as accurate in predicting colors due to the missing line-blanketing. The inclusion or not of the predicted lines has a clear impact on the colors predicted by SP models as shown in Fig. 12 by [6]. In order to provide accurate predictions for both high spectral resolution features and broad-band colors, either libraries that do not include the predicted lines must be flux calibrated [e.g. section 3.2 in 6], or low and high resolution SP models should be computed with different libraries [as adopted by 46].

\section{A NOTE ON ATMOSPHERIC PARAMETERS}

Accurate atmospheric parameters $-\mathrm{T}_{\mathrm{eff}}, \log g$ and abundances - are essential to link the stars from the spectral library to stellar evolution predictions, the other crucial ingredient of a SP model. In one hand, quite often the parameters of observed stellar spectra are derived by comparison to models, or to calibrations which are largely based on models [e.g. 20]. On the other hand, modelers of stellar spectra need stars with $\mathrm{T}_{\text {eff }}$ and $\log$ $g$ derived by fundamental ways (independent or weakly dependent on models, see e.g. [47]) in order to test and calibrate the models. In the case of temperatures, for example, direct estimation of $\mathrm{T}_{\mathrm{eff}}$ is possible for close stars if the angular diameter of a star is known (interferometric measurements or lunar occultations) [e.g. 48, 49]. Recent determinations are able to determine $\mathrm{T}_{\text {eff }}$ with a typical accuracy of $5 \%$ (better for well studied stars such as Arcturus, see e.g. compilations in [50, 21]), placing a lower limit in the absolute accuracy we can obtain for $\mathrm{T}_{\text {eff }}$. Moreover, bellow $\sim 3400 \mathrm{~K}$ most (if not all) giants are variable, and we may wonder what published values of $\mathrm{T}_{\text {eff }}$ and $\log g$ for those stars really mean.

In the case of empirical libraries, methods of deriving the atmospheric parameters based on a reference sample of well studied stars [e.g. TGMET: 51, 52] are crucial to guarantee homogeneous estimations, and were indeed adopted by e.g. [11, 14]. It does not guarantee, however, against systematic errors, if the parameters of the reference stars are affected by undetected systematic deviations. Moreover, the reference stars usually encompass a limited range of spectral types, and outside this range the derived parameters are less reliable. In the case of theoretical libraries atmospheric parameters are known by construction, but evidence show that models have room to improve yet, as discussed previously. In this context, an exercise done in [23] in very appropriate: these authors compared the high resolution spectrum of the Sun to a small grid of theoretical 

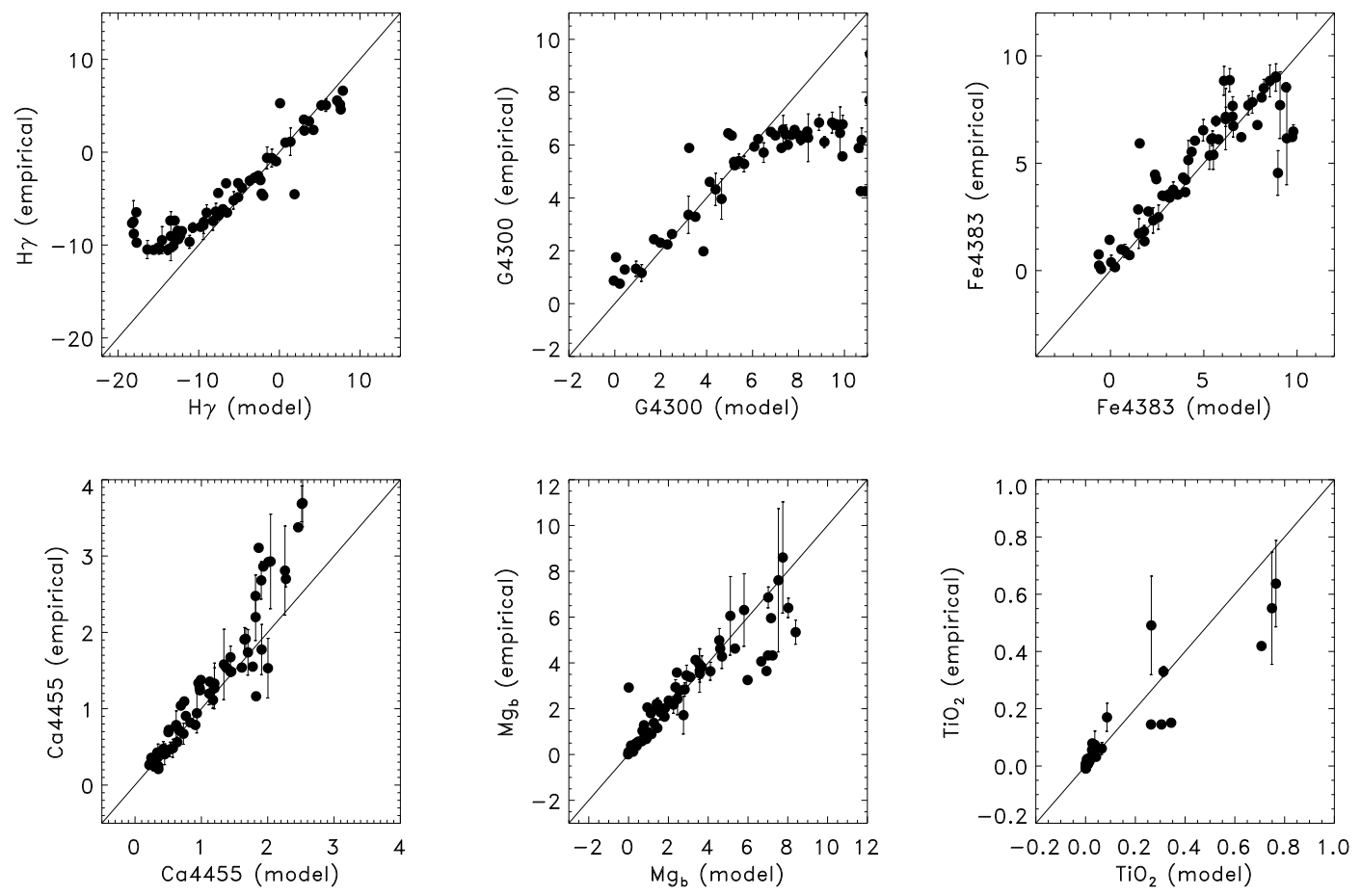

FIGURE 2. Comparison between predictions of the theoretical library by Coelho et al. in the x-axis and indices measured in the MILES empirical library in the y-axis, for six spectral indices indicated by the labels. Stars in the empirical libraries were binned in parameter space with $\Delta \mathrm{T}_{\text {eff }}=250 \mathrm{~K}$ and $\Delta \log g=0.5$ dex (reasonable values given the atmospheric parameters uncertainties). Mean values in each parameter bin were plotted against the theoretical predictions, and error bars illustrate the 1 sigma deviations. Points without error bars occur when only one star in the empirical library falls into a parameter bin. The solid line is the one-to-one relation.

libraries, and derived the solar parameters using the theoretical grid as reference stars. The parameters derived for the Sun have offsets with respect to the real values of $\Delta \mathrm{T}_{\mathrm{eff}}=$ $+80 \mathrm{~K}, \Delta \log g=+0.5$ and $\Delta[\mathrm{Fe} / \mathrm{H}]=-0.3$. In a sense, these offsets quantify the accuracy of the theoretical libraries in a scale that can be directly compared to the uncertainties of the atmospheric parameters in empirical libraries.

In any case, the impact of (realistic) errors of the atmospheric parameters on the predictions of SP models remains to be quantified.

\section{CONCLUSIONS}

Empirical stellar libraries are mature, and are the most reliable for the modeling of SP in the optical and near-IR wavelengths. The coverage in terms of $\mathrm{T}_{\text {eff }}$ and $\log g$ is very good around solar metallicities. Outside the solar metaliticy regime, the range of SP ages that can be modeled is somewhat restricted. The main caveat of empirical libraries is their inability to model accurately populations that have undergone a star formation history different than the stars in the library (biased towards the solar neighborhood). This is 
an issue in interpreting large samples of medium to high spectral resolution integrated spectra of clusters and galaxies. Future advancements in empirical libraries will likely come from space telescopes, to better explore regions beyond the visible range [e.g. NGSL by 53].

Theoretical libraries are to some extend well calibrated in the visible and near-IR for stars as late as G-type. Colors in the visible and near-IR bands are reproduced within the error bars for temperatures down to $\sim 3500 \mathrm{~K}$. At a resolution of $\mathrm{R}=10^{5}$, the spectrum of the Sun is today reproduced in 5\% of relative flux, Arcturus is reproduced in $9 \%$ and Vega is reproduced in $1 \%$. Residuals are in general larger towards cooler stars or lower surface gravities. For stars below $\sim 3500 \mathrm{~K}$, current developments in hydrodynamical models and pulsating atmospheres should improve the accuracy of the models, but it may well take some years before such grids are available to the completeness needed for population synthesis. For O- and B-type stars, recent developments in mass loss modeling, expanding atmospheres and wind features are being incorporated into the theoretical grids to model the UV with better accuracy.

Theoretical (or possibly semi-theoretical) libraries are the most promising to model the integrated spectral features of populations beyond the local one. As the observations of extra-galactic populations improve in terms of spectral resolution, environment and redshift coverage, it is my personal view that next generations of libraries for SP modeling will tend more and more towards theoretical libraries, with empirical libraries being used for the crucial testing and calibration of the theoretical spectra.

Acknowledgments: The author acknowledges the support of the European Community under a Marie Curie International Incoming Fellowship (6th Framework Programme, FP6).

\section{REFERENCES}

1. B. M. Tinsley, Fundamentals of Cosmic Physics 5, 287-388 (1980).

2. A. Vazdekis, ApJ 513, 224-241 (1999).

3. D. Le Borgne, B. Rocca-Volmerange, P. Prugniel, A. Lançon, M. Fioc, and C. Soubiran, $A \& A 425$, 881-897 (2004).

4. G. Bruzual, and S. Charlot, MNRAS 344, 1000-1028 (2003).

5. R. M. G. Delgado, M. Cerviño, L. P. Martins, C. Leitherer, and P. H. Hauschildt, MNRAS 357, 945-960 (2005).

6. P. Coelho, G. Bruzual, S. Charlot, A. Weiss, B. Barbuy, and J. W. Ferguson, MNRAS 382, 498-514 (2007).

7. J. K. Adelman-McCarthy, M. A. Agüeros, S. S. Allam, and et al., ApJS 175, 297-313 (2008).

8. J.-F. Le Borgne, G. Bruzual, R. Pelló, A. Lançon, B. Rocca-Volmerange, B. Sanahuja, D. Schaerer, C. Soubiran, and R. Vílchez-Gómez, A\&A 402, 433-442 (2003).

9. E. Jehin, S. Bagnulo, C. Melo, C. Ledoux, and R. Cabanac, "The UVES Paranal Observatory Project: a public library of high resolution stellar spectra," in IAU Symposium, edited by V. Hill, P. François, and F. Primas, 2005, pp. 261-262.

10. F. Valdes, R. Gupta, J. A. Rose, H. P. Singh, and D. J. Bell, ApJS 152, 251-259 (2004).

11. P. Prugniel, and C. Soubiran, A\&A 369, 1048-1057 (2001).

12. P. Prugniel, C. Soubiran, M. Koleva, and D. Le Borgne, ArXiv Astrophysics (2007), arXiv:astro-ph/0703658

13. P. Sánchez-Blázquez, R. F. Peletier, J. Jiménez-Vicente, N. Cardiel, A. J. Cenarro, J. Falcón-Barroso, J. Gorgas, S. Selam, and A. Vazdekis, MNRAS 371, 703-718 (2006). 
14. A. J. Cenarro, R. F. Peletier, P. Sánchez-Blázquez, S. O. Selam, E. Toloba, N. Cardiel, J. FalcónBarroso, J. Gorgas, J. Jiménez-Vicente, and A. Vazdekis, MNRAS 374, 664-690 (2007).

15. A. Pietrinferni, S. Cassisi, M. Salaris, and F. Castelli, ApJ 612, 168-190 (2004).

16. A. Lançon, and M. Mouhcine, $A \& A$ 393, 167-181 (2002).

17. G. Worthey, S. M. Faber, and J. J. Gonzalez, ApJ 398, 69-73 (1992).

18. A. McWilliam, ARA\&A 35, 503-556 (1997).

19. P. Coelho, "Model stars for the modelling of galaxies: alpha-enhancement in stellar populations models," in Proceedings of "XII Reunion Latinoamericana (2007)", RevMexAA, in press, arXiv:astro-ph/0802.2665.

20. M. S. Bessell, F. Castelli, and B. Plez, A\&A 333, 231-250 (1998).

21. A. Kučinskas, P. H. Hauschildt, H.-G. Ludwig, I. Brott, V. Vansevičius, L. Lindegren, T. Tanabé, and F. Allard, A\&A 442, 281-308 (2005).

22. L. P. Martins, and P. Coelho, MNRAS 381, 1329-1346 (2007).

23. E. Bertone, A. Buzzoni, M. Chávez, and L. H. Rodríguez-Merino, A\&A 485, 823-835 (2008).

24. P. Coelho, B. Barbuy, J. Melendez, R. Schiavon, and B. Castilho, A\&A 443, 735 (2005).

25. F. Allard, T. Guillot, H.-G. Ludwig, P. H. Hauschildt, A. Schweitzer, D. R. Alexander, and J. W. Ferguson, "Model Atmospheres and Spectra: The Role of Dust," in Brown Dwarfs, edited by E. Martín, 2003, vol. 211 of IAU Symposium, pp. 325.

26. R. Collet, M. Asplund, and F. Thévenin, $A \& A$ 442, 643-650 (2005).

27. A. Lançon, P. H. Hauschildt, D. Ladjal, and M. Mouhcine, A\&A 468, 205-220 (2007).

28. H. J. Muthsam, B. Löw-Baselli, C. Obertscheider, M. Langer, P. Lenz, and F. Kupka, MNRAS 380, 1335-1340 (2007).

29. B. Freytag, and S. Höfner, $A \& A$ 483, 571-583 (2008).

30. T. Lanz, and I. Hubeny, ApJS 146, 417-441 (2003).

31. T. Lanz, and I. Hubeny, ApJS 169, 83-104 (2007).

32. L. Martins, R. Delgado, C. Leitherer, M. Cerviño, and P. Hauschildt, MNRAS 358, 49-65 (2005).

33. A. W. A. Pauldrach, T. L. Hoffmann, and M. Lennon, A\&A 375, 161-195 (2001).

34. A. J. van Marle, S. P. Owocki, and N. J. Shaviv, MNRAS 389, 1353-1359 (2008).

35. R. L. Kurucz, "Including all the Lines," in Radiative Transfer and Applications to Very Large Telescopes, edited by P. Stee, 2006, vol. 18 of EAS Publications Series, pp. 129-155.

36. J. Meléndez, and B. Barbuy, ApJS 124, 527-546 (1999).

37. J. Fuhr, and W. Wiese, Journal of Physical and Chemical Reference Data 35, 1669-1809 (2006).

38. W. Wiese, and J. Fuhr, Journal of Physical and Chemical Reference Data 36, 1287-1345 (2007).

39. R. L. Kurucz, "An Atomic and Molecular Data Bank for Stellar Spectroscopy," in Laboratory and Astronomical High Resolution Spectra, edited by A. J. Sauval, R. Blomme, and N. Grevesse, 1995, vol. 81 of Astronomical Society of the Pacific Conference Series, pp. 583.

40. P. Westera, T. Lejeune, R. Buser, F. Cuisinier, and G. Bruzual, A\&A 381, 524-538 (2002).

41. F. Castelli, and R. L. Kurucz, "New Grids of ATLAS9 Model Atmospheres," in Modelling of Stellar Atmospheres, IAU Symp 210, Astronomical Society of the Pacific, 2003, p. A20.

42. I. Brott, and P. H. Hauschildt, "A PHOENIX Model Atmosphere Grid for Gaia," in The ThreeDimensional Universe with Gaia, edited by C. Turon, K. S. O'Flaherty, and M. A. C. Perryman, 2005, vol. 576 of ESA Special Publication, p. 565.

43. B. Gustafsson, B. Edvardsson, K. Eriksson, U. G. Jørgensen, Å. Nordlund, and B. Plez, A\&A 486, 951-970 (2008).

44. R. C. Peterson, B. Dorman, and R. T. Rood, ApJ 559, 372-387 (2001).

45. L. H. Rodríguez-Merino, M. Chavez, E. Bertone, and A. Buzzoni, ApJ 626, 411-424 (2005).

46. S. M. Percival, M. Salaris, S. Cassisi, and A. Pietrinferni, ApJ 690, 427-439 (2009).

47. R. Cayrel, "Determination of Fundamental Parameters," in Observed HR Diagrams and Stellar Evolution, edited by T. Lejeune, and J. Fernandes, 2002, vol. 274 of Astronomical Society of the Pacific Conference Series, pp. 133.

48. A. D. Code, R. C. Bless, J. Davis, and R. H. Brown, ApJ 203, 417-434 (1976).

49. G. P. di Benedetto, $A \& A$ 270, 315-334 (1993).

50. M. Jerzykiewicz, and J. Molenda-Zakowicz, Acta Astronomica 50, 369-380 (2000).

51. D. Katz, C. Soubiran, R. Cayrel, M. Adda, and R. Cautain, A\&A 338, 151-160 (1998).

52. C. Soubiran, D. Katz, and R. Cayrel, $A \& A S$ 133, 221-226 (1998).

53. M. D. Gregg, H. C. Ferguson, D. Minniti, N. Tanvir, and R. Catchpole, AJ 127, 1441-1459 (2004). 